\title{
Simulation Analysis and Model of Current Retrieval Based on Marine Radar Sea Clutter Images
}

\author{
Liqiang Liu, ${ }^{1}$ Yuntao Dai, ${ }^{2}$ and Jinyu Gao ${ }^{1}$ \\ ${ }^{1}$ College of Automation, Harbin Engineering University, 145 Nantong Street, Heilongjiang 150001, China \\ ${ }^{2}$ College of Science, Harbin Engineering University, 145 Nantong Street, Heilongjiang 150001, China \\ Correspondence should be addressed to Liqiang Liu; 1lq9842222@126.com
}

Received 12 December 2013; Accepted 7 February 2014; Published 24 March 2014

Academic Editor: Xiaojie Su

Copyright (c) 2014 Liqiang Liu et al. This is an open access article distributed under the Creative Commons Attribution License, which permits unrestricted use, distribution, and reproduction in any medium, provided the original work is properly cited.

\begin{abstract}
Using the sea clutter image from X-Band radar for current retrieval is an effective way of obtaining information on ocean currents. Traditional methods used for current retrieval have been based on the least squares algorithm, which is not only simple and efficient but also generally speaking accurate. In order to improve the precision of current retrieval, this paper has, as its goal, the study of the used radar connected with sea clutter imaging for current retrieval, with the particle swarm optimization (PSO) algorithm being proposed. This method is achieved by obtaining a three-dimensional image spectrum, taking the high-order dispersion relation model as the theoretical distribution model of the wave energy points of three-dimensional image spectra, using a forward model within the PSO framework, and considering the requirements of the order of the model, weights and optimal distribution of the energy points, and so on in fitness function. Simulation results show that, compared with the traditional ILSM methods, the method provided in this paper is more flexible, with a capacity for a high dispersion relationship order, higher precision, and an increased stability in terms of current inversion.
\end{abstract}

\section{Introduction}

Ocean currents are the result of a variety of physical effects arising from a relatively stable large-scale flow. Ocean currents have a very close relationship for marine exploration, marine development, and marine navigation safety. Existing methods for obtaining the sea surface current include moored wave buoys, an analysis of stereo images, and analyses of satellite altimetry data and marine radar images. Although buoys provide reliable measurements, they are easily subject to damage and loss. The method for collecting stereo images from synthetic aperture radar is costly and time-consuming. Furthermore, orbiting satellites cannot obtain continuous data around a specified zone [1-3]. The marine radar of current telemetry is an effective means of obtaining currents. Compared with other methods, it has the advantages of wide range of detection, high safety, all-day observation, convenience, and the low price.

Current retrieval based on marine radar related to sea clutter images is essentially an optimization problem, with the least squares method (LSM) being used to implement the current strategy for solving the problem. In 1985, Young et al. were the first to suggest the use of marine radar sea clutter imaging for current retrieval [4]. A three-dimensional fast Fourier transformation (3-D FFT) was used to transform the time domain of the sea clutter image sequence into the frequency domain spectra of the three-dimensional image, and then the LSM was used for the current retrieval. As far as the high currents in the dispersion relation of the inversion effect were concerned, in 2001 Senet and others proposed a method for current retrieval based on the iterative least squares method (ILSM) [5], increasing the accuracy of sea surface stream retrieval, based on methods put forward in the literature on the subject [4]. In 2002, in their work concerning the effect of the three-dimensional image spectrum of the energy value of each point, Gangeskar proposed a method of current retrieval based on the three-dimensional image spectrum weighted least squares and applied the equation based on the regularization method, thus improving the precision of current retrieval [6]. In 2010, using the dispersion relation retrieval current target function, Tang proposed an iterative method for current estimation based on the 


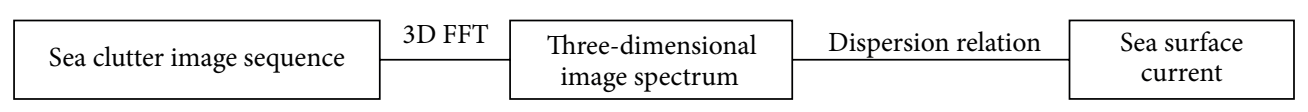

FIGURE 1: Schematic diagram illustrating current retrieval.

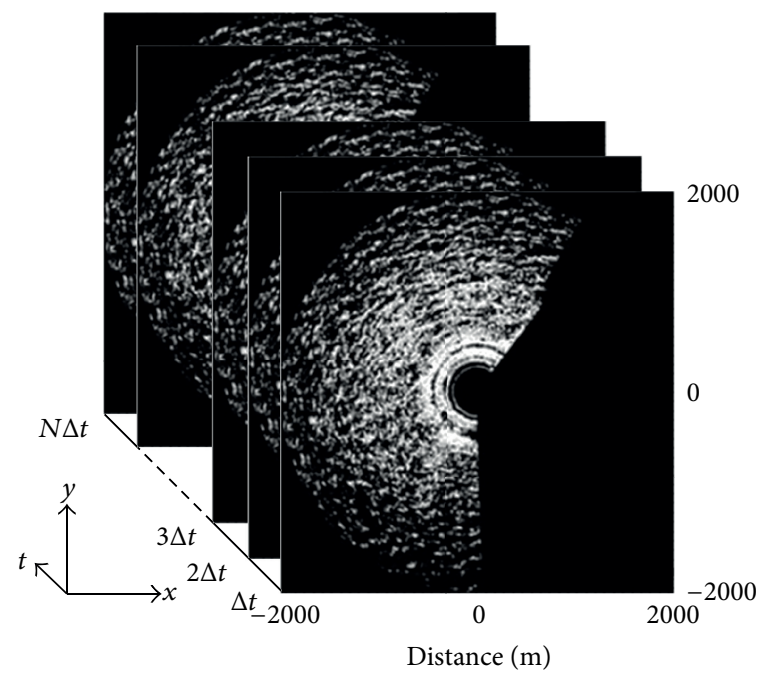

(a) Schematic diagram of sea clutter sequences

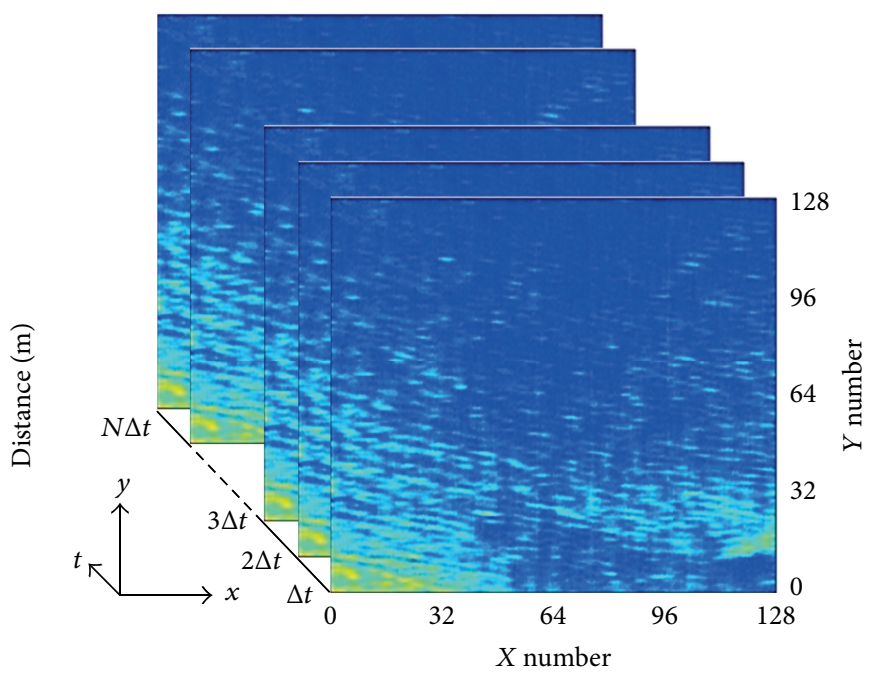

(b) Schematic illustrating rectangular analysis area of the Cartesian coordinate system

FIgURE 2: Sea clutter image data for current retrieval.

minimum variance error sequence [7] and emphasized the effect of overall energy collection on the estimated curve on the basis of the relevant literature [5]. In 2011, using the threedimensional image spectrum to extract information related to the wave spectrum as accurately as possible in order to improve the retrieval accuracy of currents, Yuan and others proposed an iterative method for estimating currents based on adaptive threshold selection [8] on the basis of the relevant literature [5].

The least squares strategy, when adopted as the method for current retrieval, has the advantage of a simple, fast solution and certainty, but, in the terms of constraint handling, processing complex optimization problems, nonlinear optimization, and so on, it has obvious shortcomings. In addition, its objective function is inflexible. In order to rectify this problem, the PSO algorithm is proposed here as a model for current retrieval. Using this algorithm, with the flexible design of this objective function, constraint condition positive expression, self-organization solving, and other characteristics for current retrieval, this model for current retrieval is discussed with a view to assess the observational data selection, the initial selection, fitness function design, process of algorithm design, and other related issues in detail. The numerical simulation results verify the correctness of the proposed method. It improves the precision and stability of the current retrieval effectively and provides a new solution for the modeling method of the current retrieval based on marine radar.

\section{Current Retrieval Fundamentals}

The method used for current retrieval based on the marine $\mathrm{X}$-Band radar related to sea clutter images was proposed by Young et al. [4] in 1985, using the Fourier transforms spectrum analysis framework, as illustrated in Figure 1.

2.1. Three-Dimensional Image Spectrum Acquisition. In order to obtain a radar three-dimensional image spectrum, threedimensional fast Fourier transformation (3-D FFT) for sea clutter images sequence continuously measured by marine radar is required, to enable us to transform the time and space domain of radar sea clutter image sequencing into the frequency domain of a radar image spectrum.

As shown in Figure 2, before the transformation operation can be performed, a rectangular analysis area from the sea clutter image sequence must be selected, and, through filtering and interpolation, the polar coordinates of the image sequence are transformed into $\eta(x, y, t)$, namely, the Cartesian coordinate system connected with grid image sequencing.

With the grid image sequence $\eta(x, y, t)$ being used for the 3-D FFT, the three-dimensional radar image spectrum $F\left(k_{x}, k_{y}, \omega\right)$ is obtained:

$$
\begin{aligned}
F & \left(k_{x}, k_{y}, \omega\right) \\
& =\int_{0}^{L_{x}} \int_{0}^{L_{y}} \int_{0}^{T} \eta(x, y, t) \cdot e^{-i\left(k_{x} \cdot x+k_{y} \cdot y-\omega \cdot t\right)} d x d y d t,
\end{aligned}
$$


where $L_{x}$ and $L_{y}$ are the rectangular analysis area, $x$ and $y$ are the direction of the spatial scale, respectively, and $T$ is the time scale of the image sequence, with $k_{x}, k_{y}$ being the wave number of $x$ and $y$, respectively, and $\omega$ the frequency. The resolutions of wave number and frequency are

$$
d k_{x}=\frac{2 \pi}{L_{x}}, \quad d k_{y}=\frac{2 \pi}{L_{y}}, \quad d \omega=\frac{2 \pi}{T} .
$$

Considering the symmetry of the Fourier transforms and in order to eliminate the $180^{\circ}$ ambiguity problems, $\omega>0$ are the only parts retained. The power spectrum density of the image is thus obtained:

$$
I\left(k_{x}, k_{y}, \omega\right)=\frac{1}{L_{x} L_{y} T}\left|F\left(k_{x}, k_{y}, \omega\right)\right|^{2} .
$$

2.2. Relation of Dispersion. Assuming that the waves satisfy the linear wave theory, homogeneous space, and stable time of the sea surface current filed in the analysis area, then when the water depth is greater than or equal to half the wavelength, a first-order approximation gravity wave will satisfy the following dispersion relation equation [9-12]:

$$
\omega_{0}(\vec{k})=\sqrt{g|\vec{k}| \tanh (d|\vec{k}|)}
$$

where $\omega_{0}$ represents the wave frequency of gravity, $\vec{k}$ represents the wave number of the wave, $d$ represents depth, and $g$ represents the acceleration of gravity.

Considering that the presence of the currents $\vec{u}$ will result in the generation of the Doppler frequency-shift, then the current $\vec{u}$ will be the same as wave number $\vec{k}$ and the angular frequency $\omega$ generating a frequency-shift affecting the image spectrum $F\left(k_{x}, k_{y}, \omega\right)$, with the dispersion relation equation as follows:

$$
S(\vec{k})=\sqrt{g|\vec{k}| \tanh (d|\vec{k}|)}+\vec{k} \cdot \vec{u} .
$$

The surface schematics of dispersion relation in various cases are shown in Figure 3.

The detection process carried out by the marine radar found that nonlinear effects are caused by the influence of sea surface imaging and the relative weakness of the sea surface waves themselves. The wave energy of the threedimensional image spectrum $F\left(k_{x}, k_{y}, \omega\right)$ of the radar exist not only in the base (level 0 ) dispersion relation equation but also in the higher-order dispersion relation equation, which is referred to as the high-order wave phenomena. The high-order dispersion relation equation can be expressed as follows:

$$
\omega_{p}=(p+1) \sqrt{\frac{g|\vec{k}|}{p+1} \cdot \tanh \left(\frac{d|\vec{k}|}{p+1}\right)}+\vec{k} \cdot \vec{u},
$$

where $p$ is the order of the dispersion relation and $\omega_{p}$ is the frequency of the wave of $p$ order.
2.3. The Basic Idea behind Current Retrieval. After $n$ sea clutter images have been measured consecutively by marine radar using the 3-D FFT, $\omega>0$ is the reserved part and the resulting spectrum of the radar image is the energy point set $I\left(k_{x}, k_{y}, \omega\right)$ distributed on the spatial grid $k_{x} \times k_{y} \times(n / 2)$. The current retrieval process involves the extraction of the energy points of the wave spectrum from the energy point collection, with (6) being used to determine the current $\vec{u}$, so that all the energy points of the wave spectrum are distributed in the parameters $\vec{u}$ of the dispersion relation surface.

\section{Current Retrieval Based on the Least Squares Algorithm}

Current retrieval was first proposed by Young et al. in 1985 in the literature [4] on the subject and was based on the average weighted least squares. All the energy points in $I\left(k_{x}, k_{y}, \omega\right)$ of this method are in line with the 0 -order dispersion relation. For any point $(\vec{k}, \omega)$, the theory of the frequency of the point $\vec{k}$ is calculated by (5). The square of the SSE of the difference between $\Delta \omega$ is

$$
\mathrm{SSE}=\sum\left(\omega-\sqrt{g|\vec{k}| \tanh (d|\vec{k}|)}-k_{x} u_{x}-k_{y} u_{y}\right)^{2} .
$$

The principle of LSM is used. The minimum of SSE is needed to find out the optimal value of $u_{x}$ and $u_{y}$ and can be obtained as follows:

$$
\begin{aligned}
& {\left[\begin{array}{l}
u_{x} \\
u_{y}
\end{array}\right]=} {\left[\begin{array}{cc}
\sum k_{x}^{2} & \sum k_{x} k_{y} \\
\sum k_{x} k_{y} & \sum k_{y}^{2}
\end{array}\right]^{-1} } \\
& \times\left[\sum(\omega-\sqrt{g|\vec{k}| \tanh (d|\vec{k}|)}) k_{x}\right] \\
&\left.\sum(\omega-\sqrt{g|\vec{k}| \tanh (d|\vec{k}|)}) k_{y}\right]
\end{aligned}
$$

In 2001, in their consideration of the impact of the higherorder dispersion relation based on the LSM method, Senet et al. proposed a current estimation method based on the Iterative Least Squares Method (ILSM) [5]. First of all, a larger threshold $C_{\mathrm{FG}}$ is selected, with the observed data $(\vec{k}, \omega)$ of spectral energy greater than $C_{\mathrm{FG}}$ being retained in $I\left(k_{x}, k_{y}, \omega\right)$ in accordance with the 0 -order dispersion relation of energy points, and the initial estimate currents $\vec{u}_{0}$ are calculated by using (8). Then the dispersion relation order $p$ included in the algorithm is determined, $\vec{u}_{0}$ is substituted, and the corresponding values of $\vec{k}$ of the order of theoretical frequency $\omega_{i}(i=0,1, \ldots, p)$ are obtained. A threshold value $C_{\mathrm{IT}}$, which is much smaller than $C_{\mathrm{FG}}$, is then selected; the spectral energy of the energy point that is greater than $C_{\mathrm{IT}}$ is reserved in $I\left(k_{x}, k_{y}, \omega\right)$, with the observed data $(\vec{k}$ , $\left.\omega^{\prime}\right)$ being obtained. By comparing the distance of $\omega^{\prime}$ and 


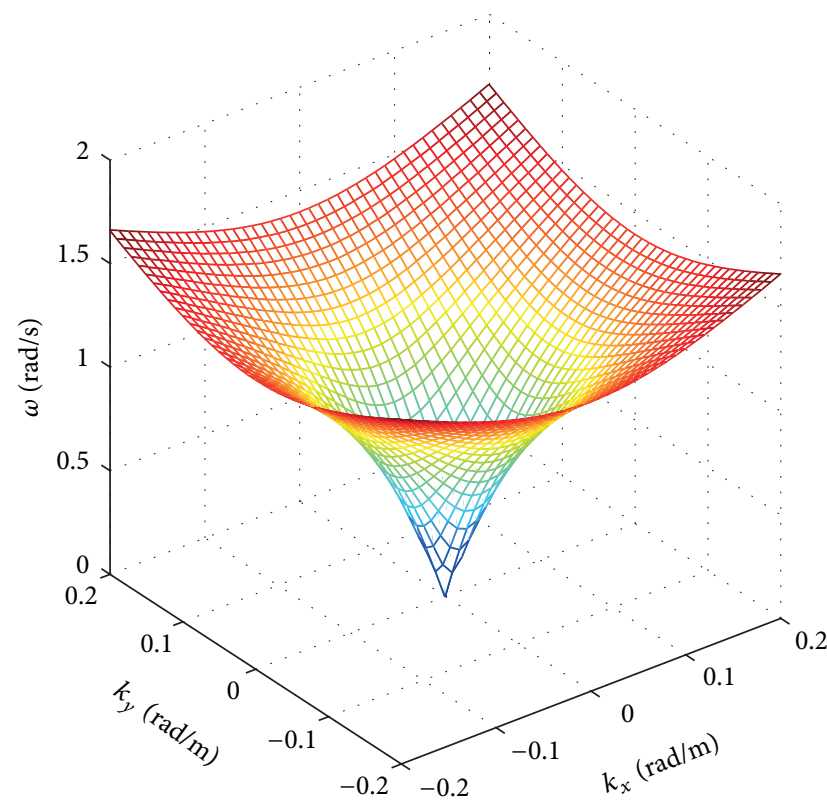

(a) Surface for velocity of $0 \mathrm{~m} / \mathrm{s}$

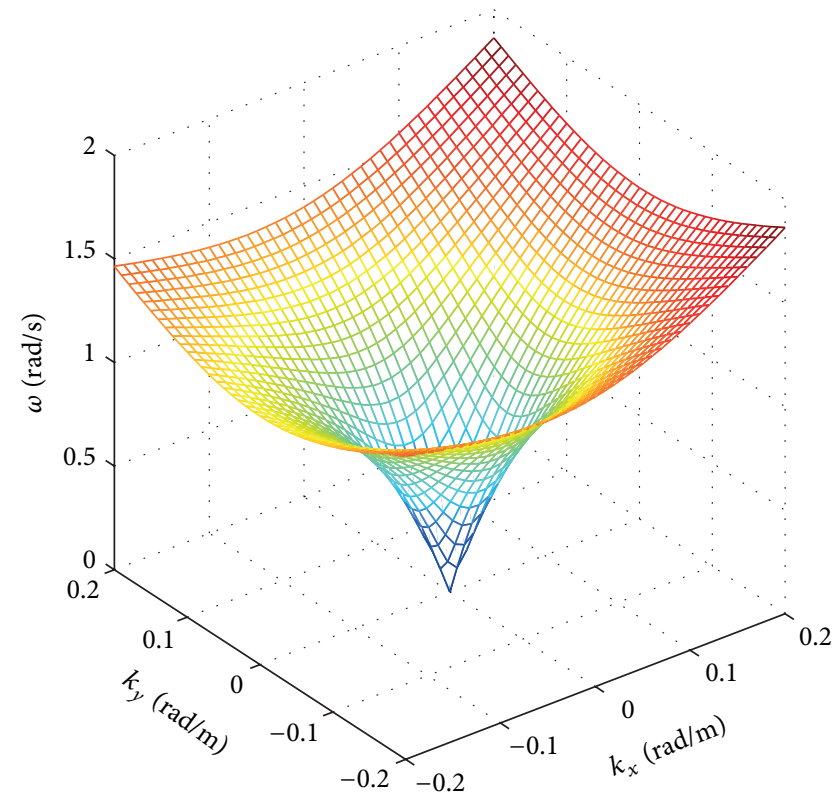

(b) Surface for velocity of $1 \mathrm{~m} / \mathrm{s}$

FIGURE 3: Surface schematics of dispersion relation.

$\omega_{i}(i=0,1, \ldots, p)$, the order of the observed data is decided. In line with the new 0 -order dispersion relation of energy points, current $\overrightarrow{u_{0}^{\prime}}$ is calculated by using (8); the above process is then repeated, with the current being calculated by iteration.

In 2002, Gangeskar put forward the weighted least squares method [6] for currents estimation based on the radar three-dimensional image spectrum considering the influence of the power spectrum on the basis of the LSM method. The objective function pulls in the spectral energy $I\left(k_{x}, k_{y}, \omega\right)$ as shown in $(7)$, so that

$$
\mathrm{SSE}=\sum I \cdot\left(\omega-\sqrt{g|\vec{k}| \tanh (d|\vec{k}|)}-k_{x} u_{x}-k_{y} u_{y}\right)^{2} .
$$

The LSM used to obtain the current estimation value is

$$
\begin{aligned}
{\left[\begin{array}{l}
u_{x} \\
u_{y}
\end{array}\right]=} & {\left[\begin{array}{cc}
\sum I k_{x}^{2} & \sum I k_{x} k_{y} \\
\sum I k_{x} k_{y} & \sum I k_{y}^{2}
\end{array}\right]^{-1} } \\
& \times\left[\sum I k_{x}(\omega-\sqrt{g|\vec{k}| \tanh (d|\vec{k}|)})\right. \\
& {\left[\sum I k_{y}(\omega-\sqrt{g|\vec{k}| \tanh (d|\vec{k}|)})\right] . }
\end{aligned}
$$

In 2010, Tang considered the use of the overall attributes belonging to the dispersion relation set and,by improving the objective function in the framework of the ILSM algorithm, they proposed a current retrieval method of minimum variance based on error sequence [7]. In this method, the objective function is written; thus

$$
\mathrm{SSE}=\sum(\Delta \omega-\Delta \bar{\omega})^{2}
$$

where $\Delta \omega=\omega-\sqrt{g|\vec{k}| \tanh (d|\vec{k}|)}-k_{x} u_{x}-k_{y} u_{y}, \Delta \bar{\omega}=$ $(1 /(M \cdot N)) \sum \Delta \omega$.

\section{Current Retrieval Model Based on PSO}

4.1. Observational Data Selection. Current retrieval using observational data is the point set of energy in the power spectral density $I\left(k_{x}, k_{y}, \omega\right)$. These energy points can be divided into three kinds: the wave energy points of the 0 order dispersion relation, the wave energy points of the high-order dispersion relation, and the energy points of the background noise. The energy points of background noise must be filtered out before the current retrieval is carried out, with only the energy points of the wave being retained. As far as the filtering out of background noise is concerned, three different methods are discussed below.

(1) Overall Selection. The total number of energy points in $I\left(k_{x}, k_{y}, \omega\right)$ is used in current retrieval. When background noise points represent lesser and their energy value are low, the overall energy of the noise is much less than the overall wave energy, making the selection of the total number of energy points simple and effective. When there is a lot of background noise or the energy value is high, the overall energy of the noise cannot be ignored, so that this selection method is effective in terms of current retrieval, and the ability to adapt to the retrieval method is greater. In addition, 
the selection of all the energy points extends the computation time of current retrieval.

(2) Fixed Number Selection. Assuming $I\left(k_{x}, k_{y}, \omega\right)$, the energy value of the high points corresponds to the wave energy points, with all the energy points in descending order. This is followed by the selection of a certain number of higher energy points to represent current retrieval of observational data. With this method, the observed data points are more complex. When the wave is small, the wave energy point is also less, so that it is only necessary to select a small number of high energy points. When the waves are large, the number selected must be increased or the wave energy will not be calculated.

(3) Selection of Energy Percentage. The maximum energy value of $I\left(k_{x}, k_{y}, \omega\right)$ up to a certain percentage is representative of the threshold, and all the energy points above the threshold are retained as the observational data of current retrieval. This approach assumes that, regardless of the circumstances, the energy of the noise points is lower. The highest relative energy value selected as the threshold is selected.

4.2. Initial Selection. As far as the PSO algorithm is concerned, the location of the information of particles is the optimization object of the algorithm. In this paper, for the current retrieval, the particle's position is the current component of $u_{x}$ and $u_{y}$. The particle dimensions should thus be two-dimensional.

The selection of the initial value of the position and speed has a bearing on the PSO. As far as the initial value of the position is concerned, if it is relatively close between the initial position and the distance of the optimal point, the initial position of the fitness value of the particle is high, which is easy to find the optimal solution for particles quickly, and if it is far between the initial position and the distance of the optimal point, the algorithm will increase the optimization time. For the initial value of the speed, if the initial value of the speed is much big, the particle can jump over a wide range in the search space and it is easy for these particles to exceed the permissible range. As far as the initial value of the speed is small, a particle that only moves within a small area is not conducive to global optimization. Generally, the initial value of position and speed of the actual solution are randomly selected within the permissible range.

In order to speed up the current retrieval and shorten the time of optimization as much as possible, the calculation of the value of the algorithm using the formula specified in (10) as the benchmark positions $\bar{u}_{x}$ and $\bar{u}_{y}$, as the initial position of the particle, is chosen as follows:

$$
\begin{aligned}
& u_{x i}=\bar{u}_{x}+\frac{\left|\bar{u}_{x}\right|}{2} N(0,1), \\
& u_{y i}=\bar{u}_{y}+\frac{\left|\bar{u}_{y}\right|}{2} N(0,1) .
\end{aligned}
$$

Due to the fact that the selection of the initial position has a particular directional meaning, the initial speed value can also be appropriately small selected.
4.3. Fitness Function. In the process of current retrieval, a PSO algorithm with an adaptive value function evaluates the advantages and disadvantages of the position (current component) of each particle. The design of the fitness function is, therefore, particularly important. In this paper, the algorithm fitness function design is defined as follows:

$$
\begin{aligned}
& \operatorname{Fit}_{k}\left(u_{x}, u_{y}\right) \\
& \begin{aligned}
=\sum A \cdot \min _{p} \mid \omega-(p+1) \sqrt{\frac{g|\vec{k}|}{p+1} \cdot \tanh \left(\frac{d|\vec{k}|}{p+1}\right)} \\
-k_{x} u_{x}-\left.k_{y} u_{y}\right|^{n},
\end{aligned}
\end{aligned}
$$

where $A$ is weight, $p$ is the order of the dispersion relation when the fitness value is taken into account, and $n$ is the order of the deviation. The weight of $A$ can be selected as any number greater than 0 , for example, 1 or $I\left(k_{x}, k_{y}, \omega\right)$. The dispersion relation order $p$ is associated with actual highorder effects, with generally 2 -order being taken as the highest order. The deviation order $n$ is able to adjust the deviation and weight $A$ for the influence of the fitness function.

Let us assume that the weight $A$ of each observational data point $\left(k_{x}, k_{y}, \omega\right)$ is given and remains unchanged. When $n=1$, the deviation order is able truly to reflect the deviation value effect on the fitness function; when $n>1$, it is equivalent to amplifying the effects of the deviation value on fitness function as well as being equivalent to weakening the role of weight $A$. When $n \rightarrow \infty$, it is equivalent to the weight $A=1$, so that all the retrieval results relative to the observed data points are evenly distributed in the vicinity of the surface dispersion relation. When $0<n<1$, it is equivalent to weakening the image of the deviation on the fitness function and to strengthening the role of the weight $A$. When $n \rightarrow 0$, it is equivalent to the role of the point with the greatest weight being magnified infinitely, so that the results of the current retrieval make the observational data points of the larger weights distribute evenly close to the surface of dispersion relation.

As far as the method of current retrieval is concerned, in the framework based on the LSM, the deviation coefficient of the objective function SSE is 2, which is equivalent to weakening the role of the weights.

4.4. Algorithmic Process. The process based on the current retrieval of PSO used in this paper is shown in Figure 4.

Step 1 (initialization). Set the learning factors $c_{1}, c_{2}$, the inertia weight $w$, the initial position of the particle (currents), and the speed. 


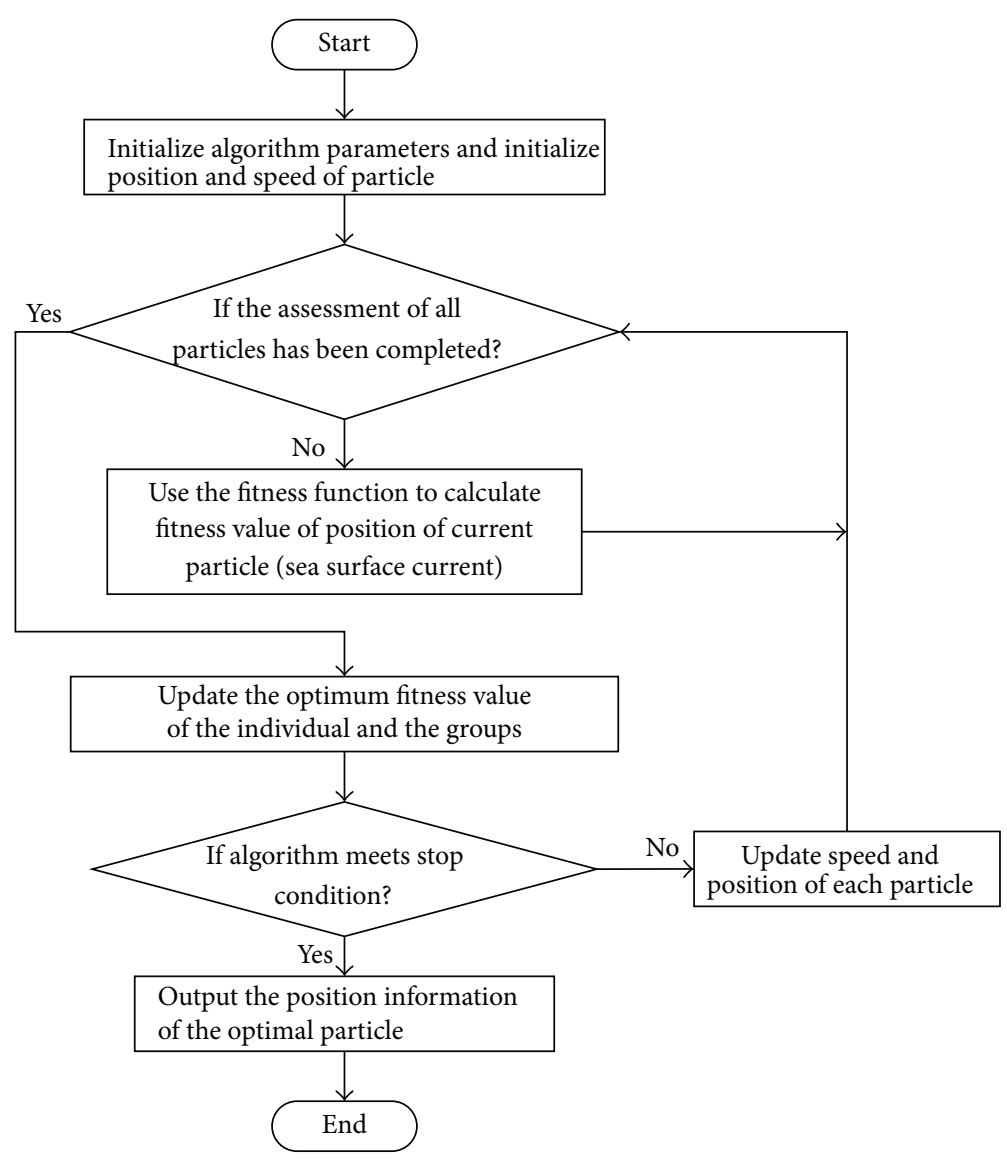

FIGURE 4: The algorithmic process.

Step 2. The fitness function is used to assess the current position of each particle to obtain the fitness value of the current position of each particle.

Step 3. The current position fitness value of each particle and the individual extreme pbest are compared and, if it is better, the fitness value is then used with the current position fitness value to update the individual extreme pbest and record the current position.

Step 4. The individual extreme value pbest of each particle is compared with the group's global extreme value gbest. If it is better, gbest is updated, and the best position recorded.

Step 5. If the termination condition is deemed to have been satisfied, then the global extremum gbest and its corresponding global positions are output; otherwise, the speed and position of the particle are updated, and "Step 2" is implemented.

\section{Simulation Analyses}

5.1. Simulation Based on Three-Dimensional Image Spectrum. Current retrieval essentially uses the energy points of a threedimensional image spectrum as observed data, according to the dispersion relation for optimal estimation. The method used for the dispersion relation used to simulate a threedimensional image spectrum is outlined as follows.

(1) Three-Dimensional Image Spectrum Simulation. It is very difficult to acquire a large number of sea clutter image sequences from marine radar and their corresponding true value. A point on a three-dimensional radar image spectrum imitatively generated based on a dispersion relation equation is proposed in this paper, and simulation experiments of current retrieval precision are carried out using the image spectrum.

Based on the previous analysis, we already know that the energy points of the three-dimensional image spectrum can be divided into two categories in line with the wave energy points of a dispersion relation and do not meet the noise energy points of the dispersion relation. These two types of energy points are therefore sufficient when the threedimensional image spectrum is generated in the simulation. This simulation takes place for a three-dimensional image spectrum according to the following principles.

Principle 1. The dispersion relation only considers three times of the 0 -order, 1 -order, and 2 -order.

Principle 2. The dispersion relation of energy points of the 0 -order is 1,1 -order is 0.2 , and 2 -order is 0.04 . 
TABLE 1: Operating parameters of PSO algorithm.

\begin{tabular}{cccccc}
\hline$m$ & $c_{1}$ & $c_{2}$ & $w_{\text {ini }}$ & $w_{\text {end }}$ & $T_{\max }$ \\
\hline 10 & 2.05 & 2.05 & 0.9 & 0.4 & 100 \\
\hline
\end{tabular}

Principle 3. The energy point standard for background noise is 0.01 , the number of wave energy points is $20 \%$, and the location is set at random.

The simulation process of the three-dimensional image spectrum is shown in Figure 5.

The formula of each energy value during the simulation of energy points of three-dimensional image spectrum is as follows:

$$
E\left(k_{x}, k_{y}, \omega\right)=\bar{E}+\frac{\bar{E}}{2} N(0,1),
$$

where $E\left(k_{x}, k_{y}, \omega\right)$ is the energy value of the point and $\bar{E}$ is energy reference value.

(2) Adaptive Ability of Dispersion Relation Order Simulation. The three-dimensional images spectrum is generated considering 0 -order and 1-order of dispersion relation by using the above method. Within the three-dimensional image spectrum, the current speed changes from $0 \mathrm{~m} / \mathrm{s}$ to $6 \mathrm{~m} / \mathrm{s}$, while the current direction changes from 0 to $2 \pi$; then the current speed changes from $6 \mathrm{~m} / \mathrm{s}$ to $0 \mathrm{~m} / \mathrm{s}$, while the current direction changes from 0 back to $2 \pi$ again. 32 groups of corresponding data points are generated.

Both the ILSM method and the method proposed here (using the PSO representation) are used for current retrieval. Among the three cases of the highest order 0,1 , and 2 of dispersion relations are, respectively, taken into consideration using the ILSM method, and, in this paper, 2-order is only considered as being the highest order. The solutions of wave power point distribution on the different orders of dispersion in three-dimensional image spectrum are counted in different simulation experiments.

150 maximum energy points from the three-dimensional image spectrum are selected as the observed data on which to carry out the simulation experiments. If the difference of current speed is continuous less than 0.1 two times, the ILSM method will be stopped. When the current speed of optimal particles remains unchanged for consecutive 10 times, the PSO method will be stopped. In the PSO algorithm, $X_{d}^{\max }$ is taken as $5, V_{d}^{\max }$ is taken as 2 , and the deviation order $n$ is 1 in the fitness function, with the empirical parameters given in [13] being used for the other parameters. The specific values are shown in Table 1 .

Different simulation results are given in Table 2. The wave energy points of the three-dimensional image spectrum at the different orders of dispersion relation are distributed over the surface.

Table 2 shows that, although the three-dimensional image imitatively generated just contains 0 -order and 1-order, some points are still attributed to the second-order dispersion relation when ILSM method considers the highest order two. In other words, in terms of the relation of dispersion order,

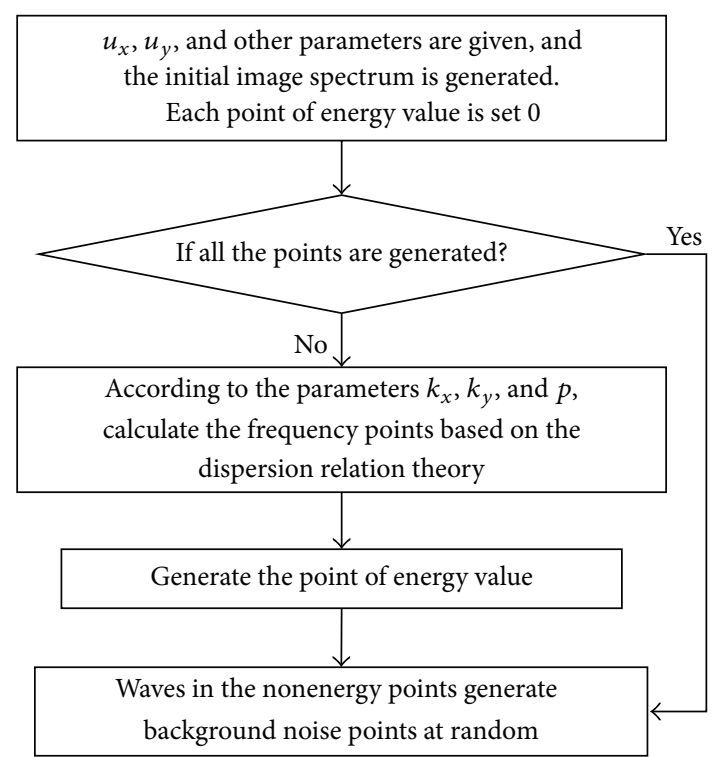

FIGURE 5: Simulation chart representing three-dimensional image spectrum.

the ILSM does not have the adaptive capacity. From the simulation results of the PSO, although the highest order of dispersion relation takes into account the second-order, the method has the adaptive ability for the order and it can identify the three-dimensional image spectrum that does not contain two order data points for the dispersion relation.

It is apparent that, when ILSM is used for current retrieval, the requested dispersion relation order should correspond to the actual dispersion relation order contained in three-dimensional image spectrum, while the order is not necessary correspondent in the PSO for the current retrieval, so that only the highest dispersion relation order needs to be set.

(3) Simulation of Precision for Current Retrieval. In this simulation, ILSM and PSO are used to simulate a threedimensional image spectrum for current retrieval by calculating the variance of current speed and current direction and evaluating the precision of the current retrieval in the two methods. The simulation methods and parameters are selected as above; the main difference is only in the following two aspects.

(a) The simulation of the three-dimensional image spectrum is taken into 2-order, with the ILSM and PSO methods also being taken into a 2-order situation.

(b) The PSO method is random. In order to evaluate the method as accurately as possible, it is necessary to count it 10 times. The mean of the 10 results and the results of 10 times for the optimum value of the standard of current speed are used to calculate the variance.

The simulation curve representing the simulation and variance of current retrieval are given in Figure 6 and Table 3 , respectively. 

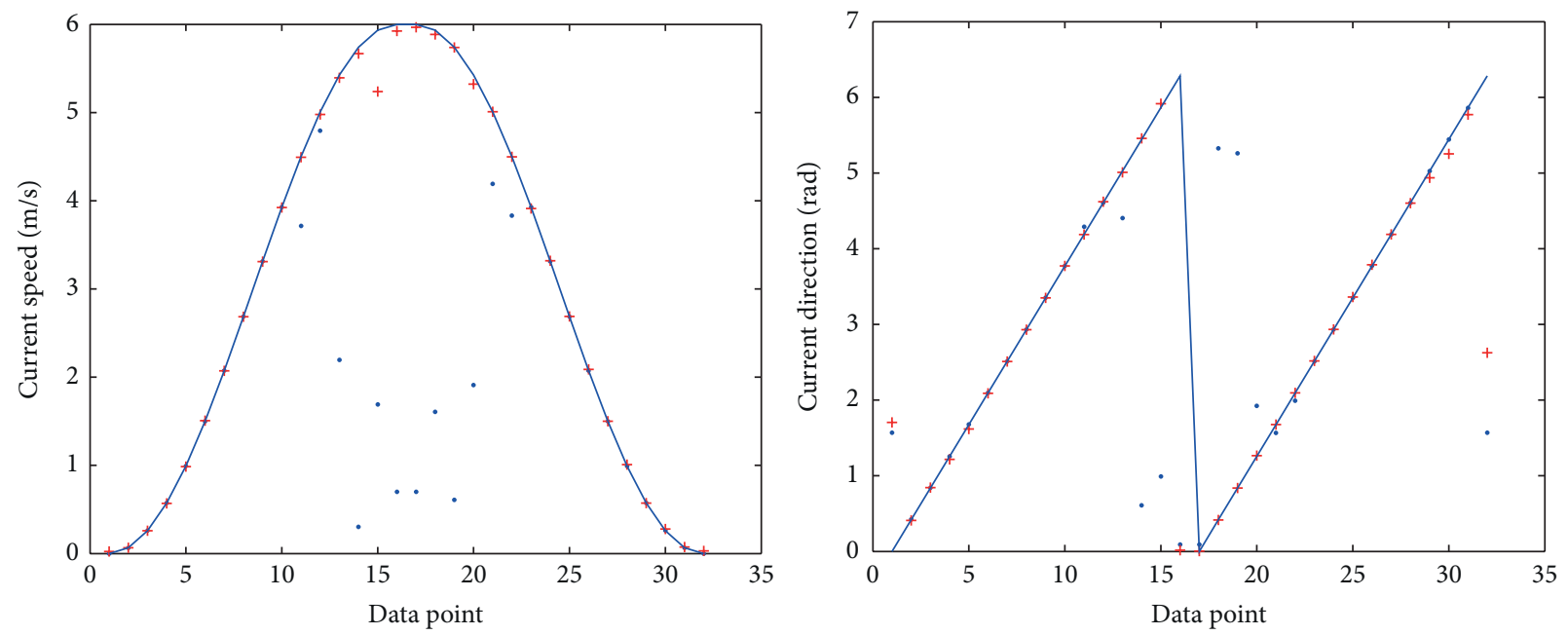

(a) Means of the results using PSO method for 10 times
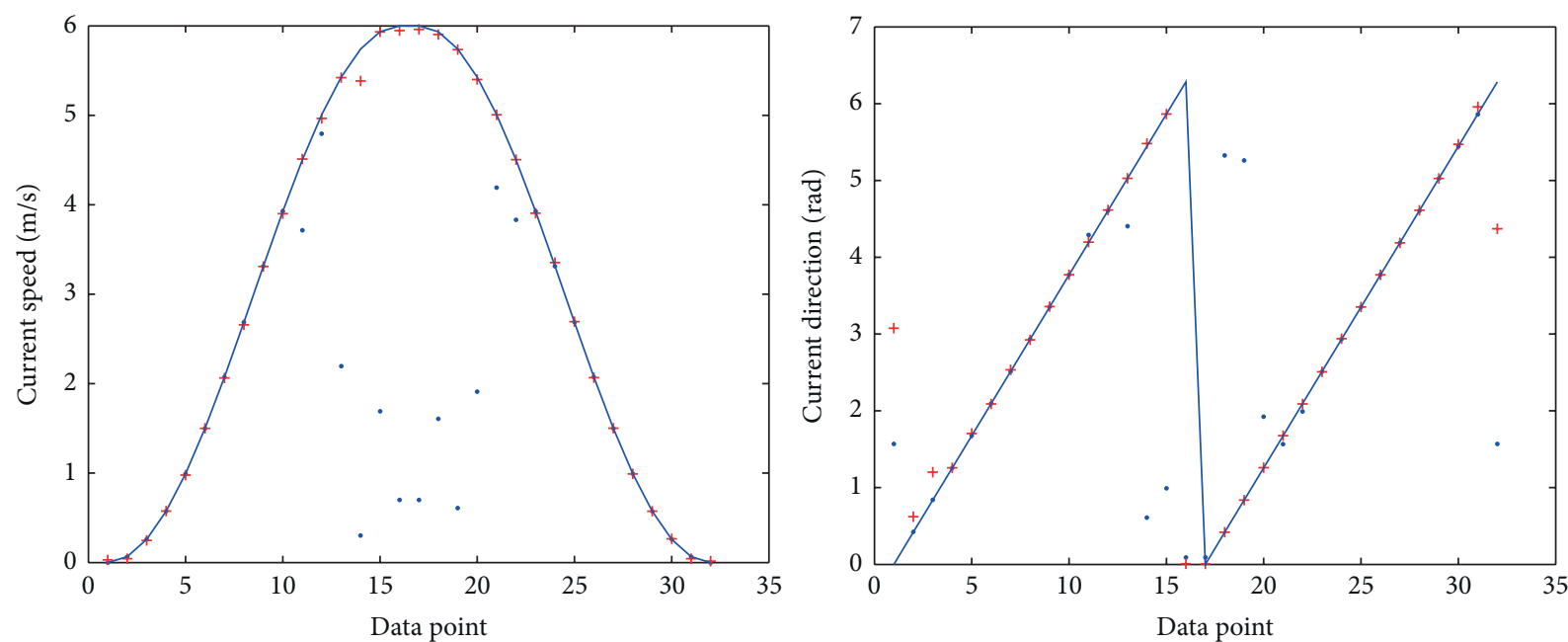

- ILSM

- ILSM

+ PSO

+ PSO

- True

- True

(b) Optimal value of results using the PSO method for 10 times, based on the standard of current speed

FIGURE 6: Simulation experiment results of current retrieval precision based on simulated three-dimensional image spectrum.

The results of the simulation show that, compared with the current retrieval results obtained by the ILSM method, the PSO method obtains better results, especially at current speed parameters. When the current speed is greater than $4 \mathrm{~m}$ per second, the current retrieval precision using PSO is obviously superior to the ILSM.

5.2. Simulation Based on Imitation Sea Clutter Images. The imitative radar sea clutter image sequence derived from literature [14] is provided in this section as the simulation radar data. In the process of generating imitative radar sea clutter image sequences, the method of adding current information is the same as in Section 5.1, generating 32 sets of files of sea clutter image sequences.

Due to the fact that the dispersion relationship in the three-dimensional image spectra is unknown, corresponding to simulation-generated radar sea clutter images, 0-order, 1-order, and 2-order are taken into account in the ILSM method, with only 2-order being taken into account in the PSO method. Simulation parameter selection and methods are consistent with Section 5.1. The simulation curve and current retrieval variance are given in Figure 7 and Table 4. The ILSM results are given in Figure 7 in which the case of 0 -order is considered.

The simulation results show that PSO method obtains a higher degree of precision than the ILSM method for curve retrieval of simulative radar sea clutter images.

5.3. Simulation Experiment Based on Real Sea Clutter Images. In the simulation experiment, real data from a radar sea clutter image sequence measured in Pingtan, Fujian, Haitan Island, China, on October 23, 2010, were used. 

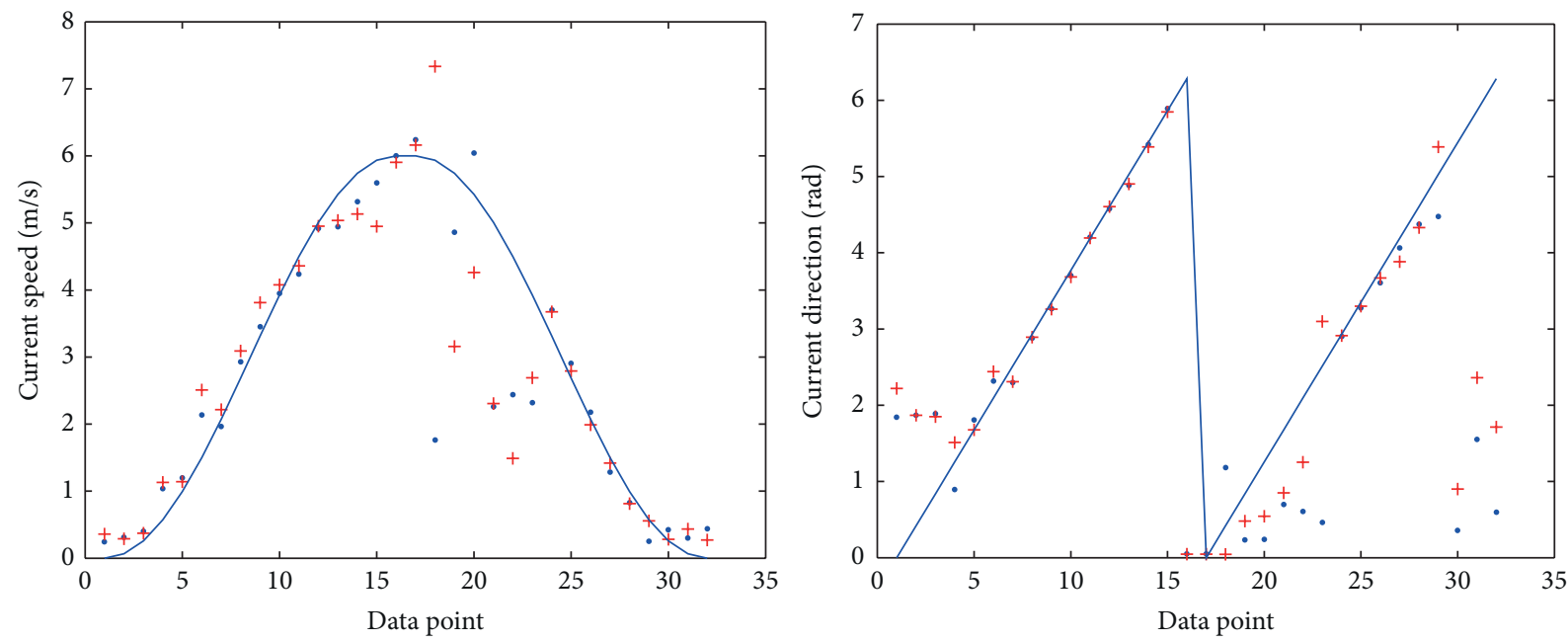

(a) Means of the results using PSO method for 10 times
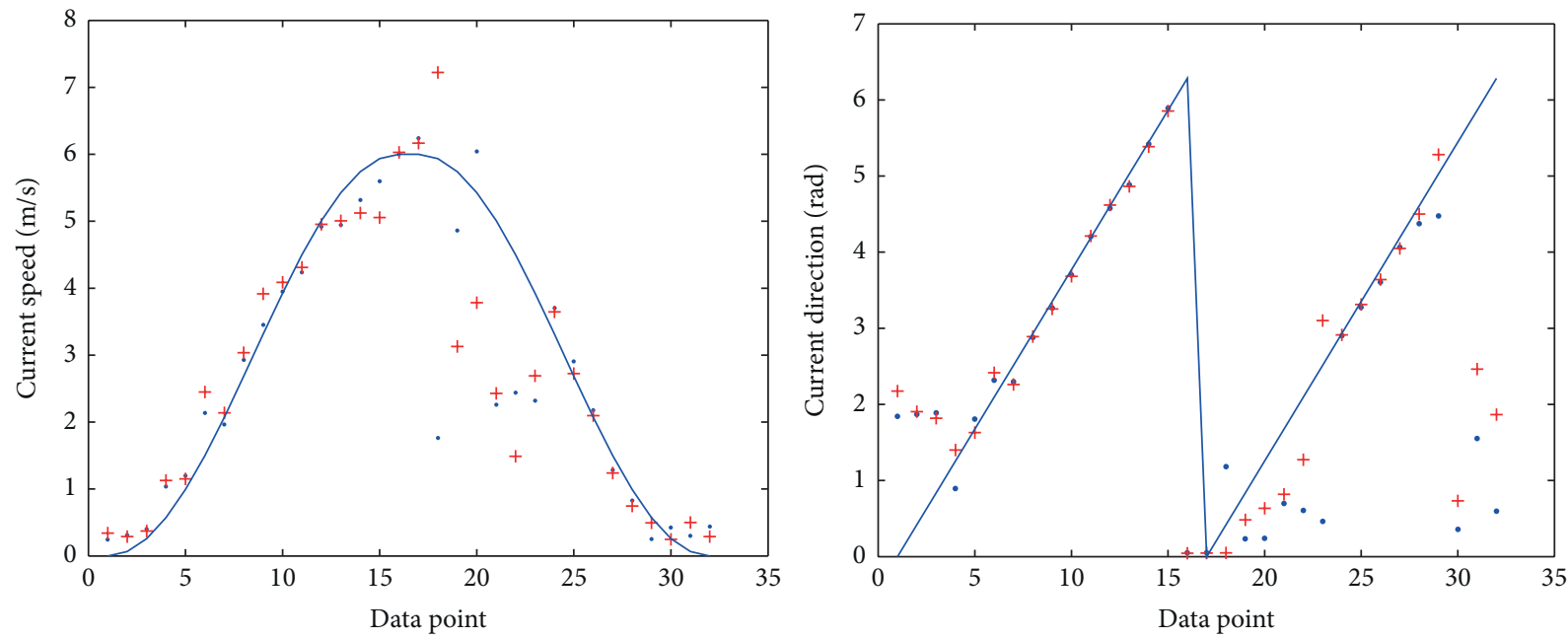

. ILSM

- ILSM

+ PSO

+ PSO

- True

- True

(b) Optimal value of results using the PSO method for 10 times based on the standard of current speed

FigURE 7: Results of simulation experimental of current retrieval precision based on imitation sea clutter images.

TABLE 2: Distributive point statistics on curve of different orders of dispersion relation.

\begin{tabular}{|c|c|c|}
\hline Method for estimated current & Order of dispersion relation & Total number of distribution points \\
\hline \multicolumn{3}{|l|}{ ILSM } \\
\hline 0-order only considered & 0 -order & 5473 \\
\hline \multirow{2}{*}{ 1-order the highest consideration } & 0 -order & 5201 \\
\hline & 1-order & 272 \\
\hline \multirow{3}{*}{ 2-order the highest consideration } & 0 -order & 5201 \\
\hline & 1-order & 233 \\
\hline & 2-order & 39 \\
\hline \multirow{3}{*}{ PSO (2-order the highest consideration) } & 0 -order & 5139 \\
\hline & 1-order & 334 \\
\hline & 2-order & 0 \\
\hline
\end{tabular}



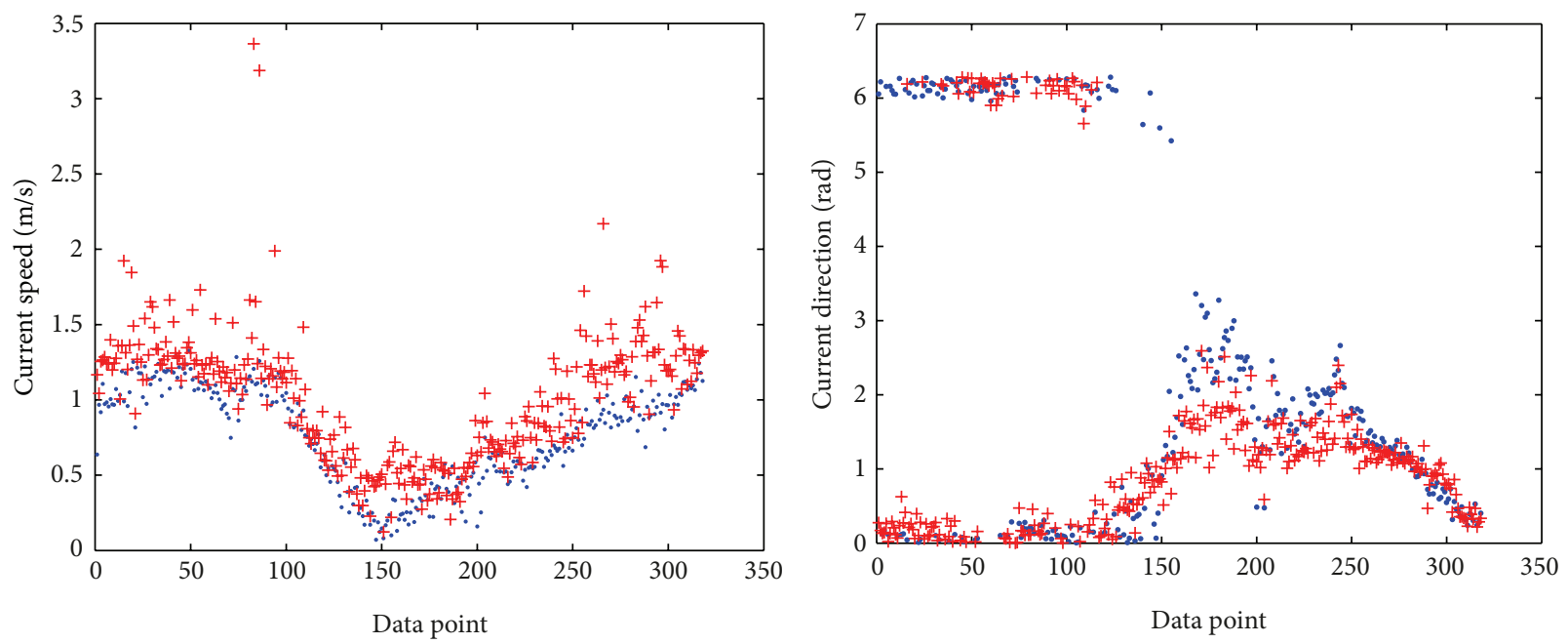

(a) 150 points of maximum energy selected
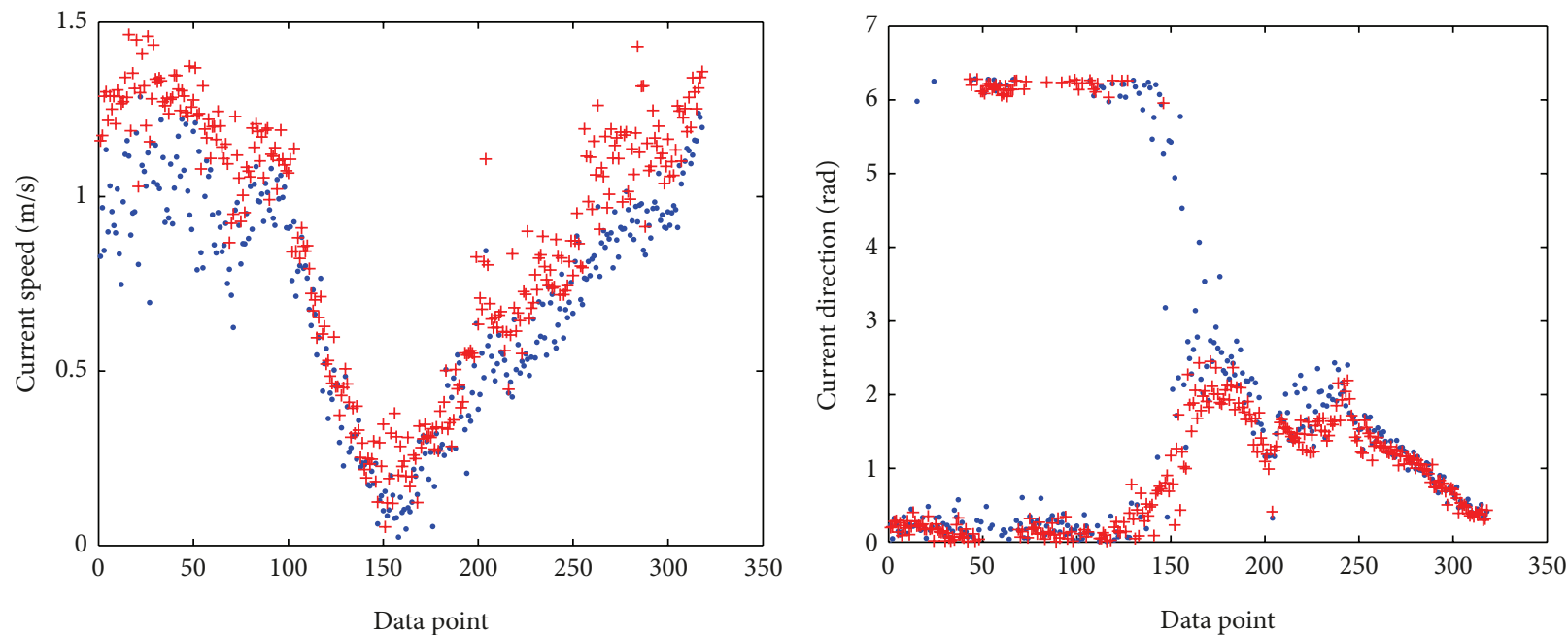

(b) 1000 points of maximum energy selected
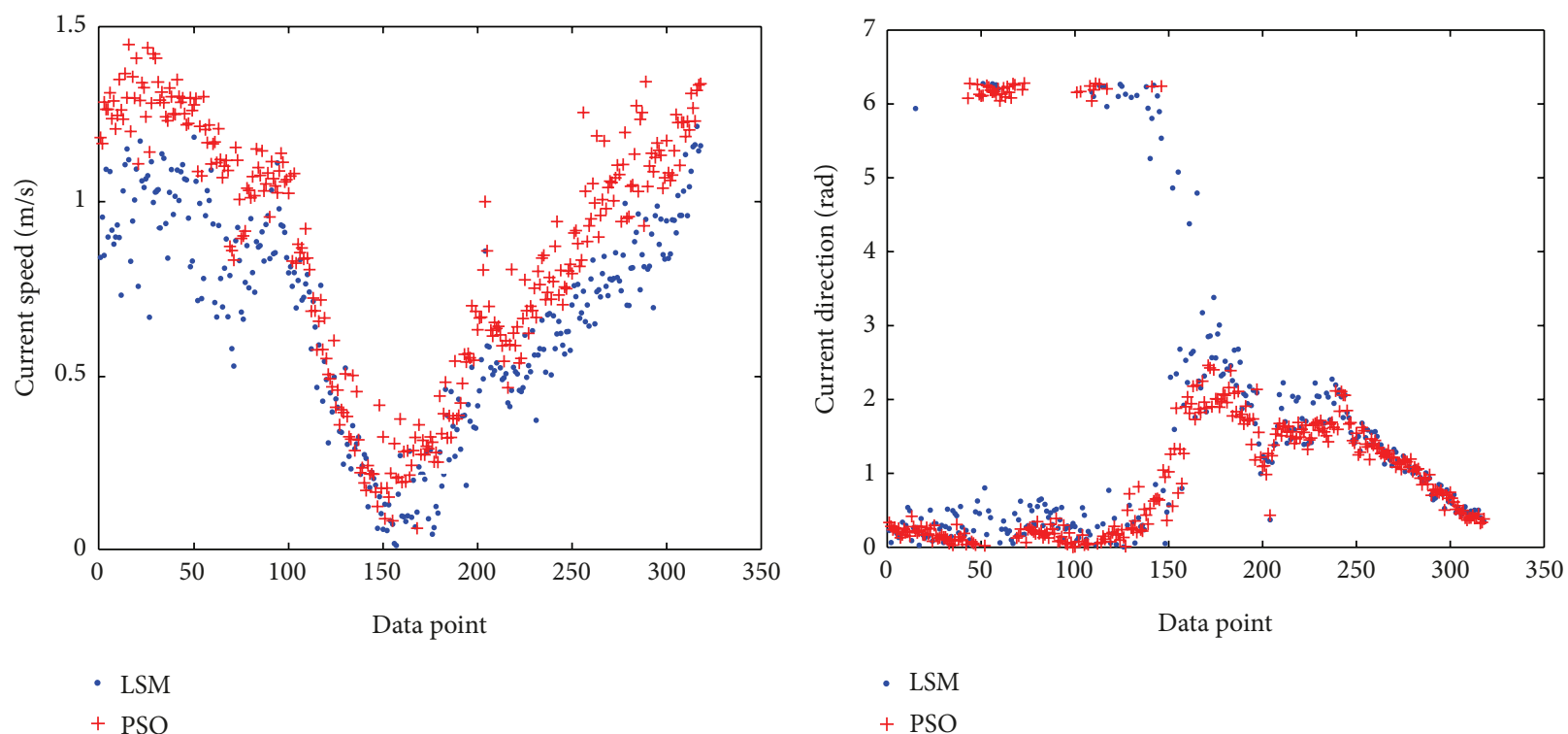

(c) Points higher than $1 \%$ of the maximum energy selected

FIGURE 8: Continued. 

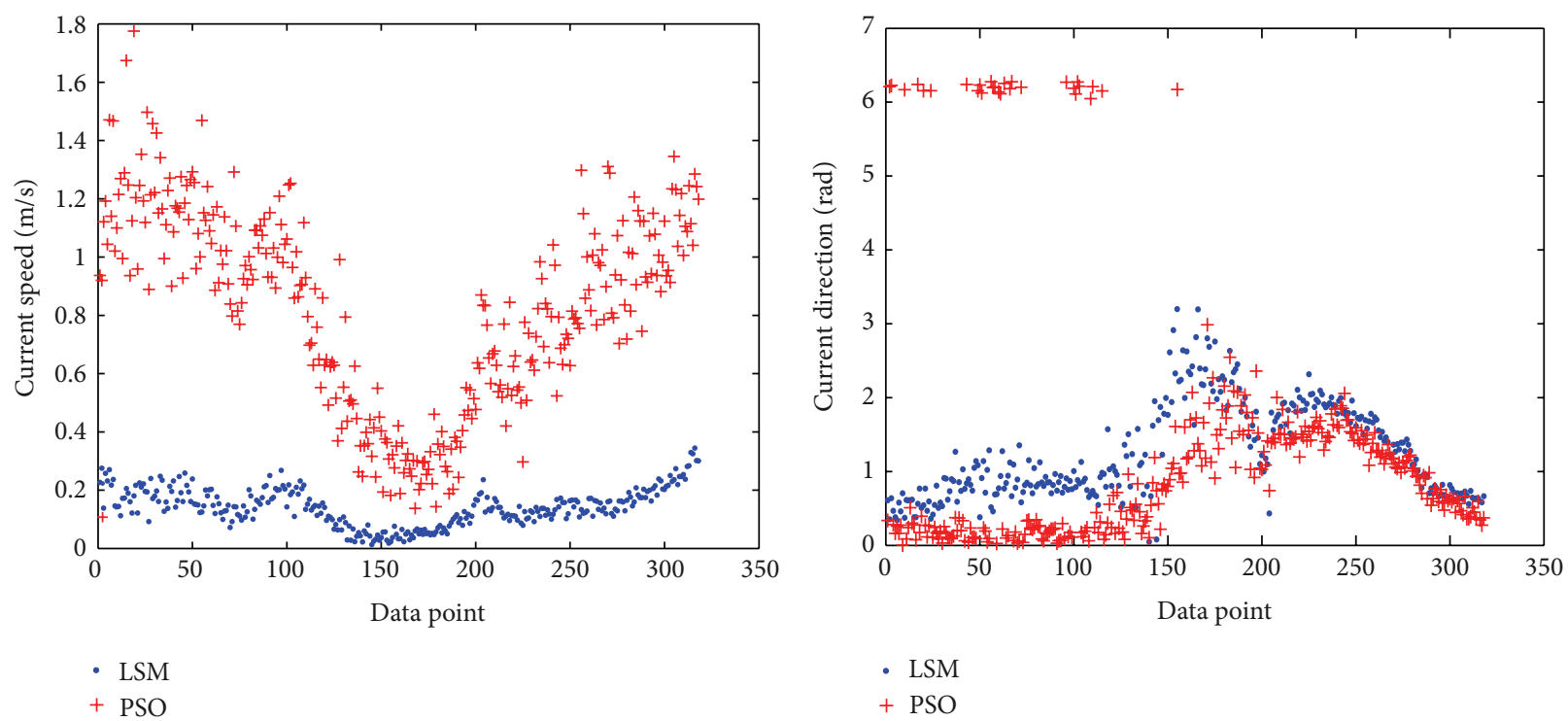

(d) All energy points selected

FIGURE 8: Simulation results of different data selection method.

TABLE 3: The variance of current retrieval based on a simulated three-dimensional image.

\begin{tabular}{lcc}
\hline Method & Current speed variance & Current direction variance \\
\hline ILSM & 2.3651 & 2.2066 \\
PSO (mean) & 0.0470 & 1.3481 \\
PSO (optimal value) & 0.0025 & 1.4861 \\
\hline
\end{tabular}

(1) Design of Evaluation Indicators. Generally speaking, the current retrieval method is assessed by comparing the error between current retrieval results and the true value. However, due to the fact that the true value of the corresponding region and currents could not be obtained from the real sea clutter image sequence from radar in the simulation experiment, the algorithms could not be properly assessed.

Consider that, for certain area current filed, its speed and directional values within the space range are uniform with small changes occurring over time. That is to say, the current changes occurring at adjacent times were small. In view of this, the mean of the differences in the continuous current retrieval results was taken as the performance evaluation indicator of the current retrieval. The calculations were made according to

$$
e=\frac{1}{n-1} \sum_{i=1}^{n-1}\left|u_{i+1}-u_{i}\right| .
$$

(2) Simulated Comparison of Dispersion Relationship Order Based on the Method of LSM. The ILSM method only considering three solutions of 0 -order, 1 -order of the highest order, and 2-order of the highest order is used for this simulation to carry out the current retrieval. The different situations related to current retrieval performance indicators are compared in order to determine the image sequence for the real sea clutter data from radar. The ILSM method should be considered the highest dispersion relationship order.
The simulated parameter selection is consistent with Section 5.1. Table 5 provides the evaluation indicator for the simulation experiment.

The ILSM method can obtain the best current estimation results which are shown in current speed indicators in Table 5, only considering the 0 -order dispersion relationship. The ILSM method is sometimes the same as the LSM method when the simulation using real radar sea clutter images sequence data contains only 0 -order dispersion relationships.

(3) Observable Data Selection Method Simulation Comparison. Simulation experiments performed on the three observational data selection methods discussed in Section 4.1 are carried out in order to evaluate the performance of the LSM and PSO methods using a different selected strategy for the observational data. The simulation curve and evaluation indicators are given in Figure 8 and Table 6.

Simulation results show that, as the selected data points increase, the current retrieval results using the PSO method are improved in terms of the polymerization and stability of the data in question. When all the data points are selected, the current retrieval is not affected by noise points, but better results are obtained. As far as the LSM method is concerned, the stability of the current retrieval results is best when 1000 points are selected. When 150 data points and higher $1 \%$ of maximum energy value are selected, current retrieval results deteriorate slightly and when all energy points are selected, current retrieval results deviate markedly from the 
TABLE 4: Inversion variance based on simulation sea clutter images.

\begin{tabular}{lcc}
\hline Method & Current speed variance & Current direction variance \\
\hline ILSM (0-order) & 1.2745 & 2.0465 \\
ILSM (1-order) & 1.0989 & 2.0656 \\
ILSM (2-order) & 1.0648 & 2.0656 \\
PSO (average) & 0.9972 & 1.8284 \\
PSO (optimal value) & 0.8553 & 1.9255 \\
\hline
\end{tabular}

TABLE 5: ILSM method evaluation indicators of different orders.

\begin{tabular}{lcc}
\hline Method & Current speed indicators & Current direction indicators \\
\hline ILSM (0-order) & 0.0937 & 0.8708 \\
ILSM (1-order) & 0.1139 & 0.6960 \\
ILSM (2-order) & 0.1165 & 0.6918 \\
\hline
\end{tabular}

true value, although an increased stability can be obtained. If we compare the PSO and LSM methods, the PSO method is slightly less stable than the LSM method when 150 points are selected, while the performance of the PSO method is better in the other cases.

(4) Initial Value Method Simulation Comparison. Simulation is carried out using the different initial values selected for the PSO method and discussed in Section 4.2. The initial value is selected using the random initialization method and the algorithm initial current speed being randomly selected with the range $[-3,3]$. When the initial current speed is selected for the LSM initialization method, the initial current speed is shown in (12). Simulation data points are selected according to the percentage of energy. Selection parameters are consistent with Section 5.1. Evaluation indicators of the simulation experiment are given in Table 7.

Evaluation indicators show that the stability of current retrieval is consistent in the two strategies with speed optimization showing that the strategy for the initial value of the LSM results is faster and the number of algorithm iterations is less.

(5) Deviation Order Selection Method Simulation Comparison. The simulation experiments are carried out using different fitness function deviation orders to evaluate the stability of the PSO method in different deviation orders. The current retrieval is simulated when $n$ is 2,1 , and 0.5 , respectively. The selected parameters are consistent with Section 5.1. The simulation curve and evaluation indicators are given in Figure 9 and Table 8 .

The simulation results show that, when deviation order value is small, the current order is more stable. That is to say, it can be beneficial to the stability of the current retrieval when there is a focus on the three-dimensional image spectrum related to large energy points.

\section{Conclusion}

This paper had, as its goal, an improvement in the accuracy of current retrieval methods, with a study concerning radar related to sea clutter images used for current retrieval. The principle of current retrieval and methods used for current retrieval based on the least squares algorithm were introduced in this paper with the PSO algorithm being proposed as a viable method for current retrieval. Observational data and the selection strategy of the position of initial particles constituted its main focus, with the fitness function of the design, taking into account the impact of a higher dispersion relationship order and providing the framework for execution of the algorithms. Simulation experiments were based on three cases related to the three-dimensional image spectrum, sea clutter images analog, and real sea clutter in order to verify several aspects of the algorithms under investigation, namely, the adaptive capacity of the order of higherorder dispersion relations, the observational data selection method, the particle initialization selection method, the order bias selection method, and the current retrieval accuracy performance. Simulation results show that, compared with the traditional ILSM methods, the method provided in this paper is more flexible, with a capacity for high dispersion relationship order, higher precision, and an increased stability in terms of current inversion.

\section{Conflict of Interests}

The authors declare that there is no conflict of interests regarding the publication of this paper.

\section{Acknowledgments}

This work was supported by the National Natural Science Foundation of China under Grant nos. 51009036, 51109041, 

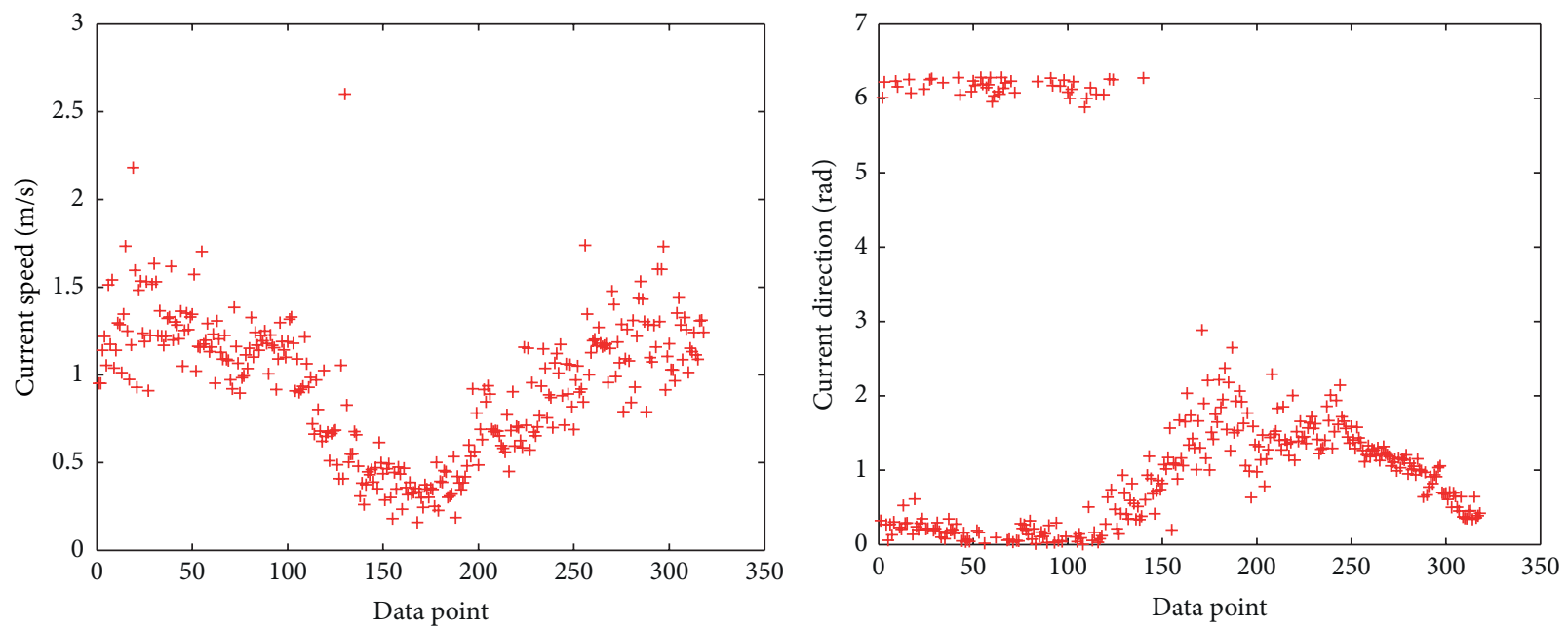

(a) $n=2$
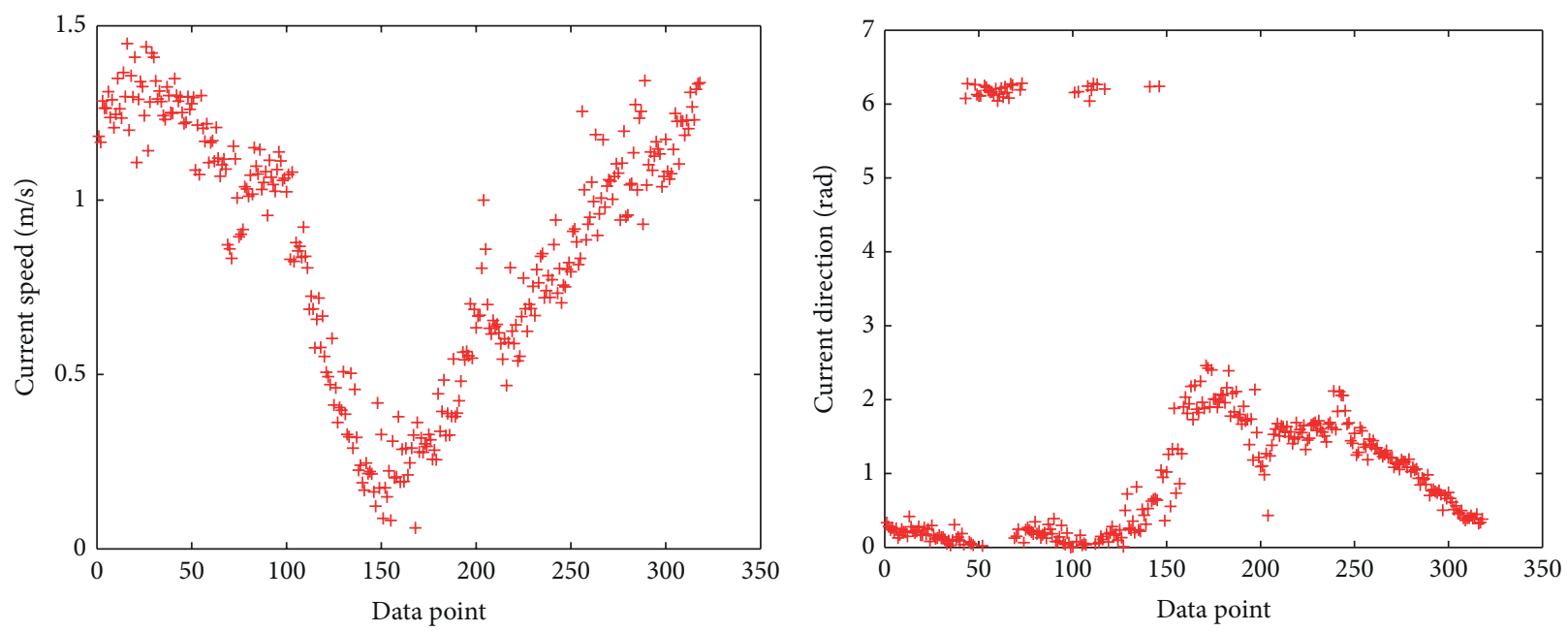

(b) $n=1$
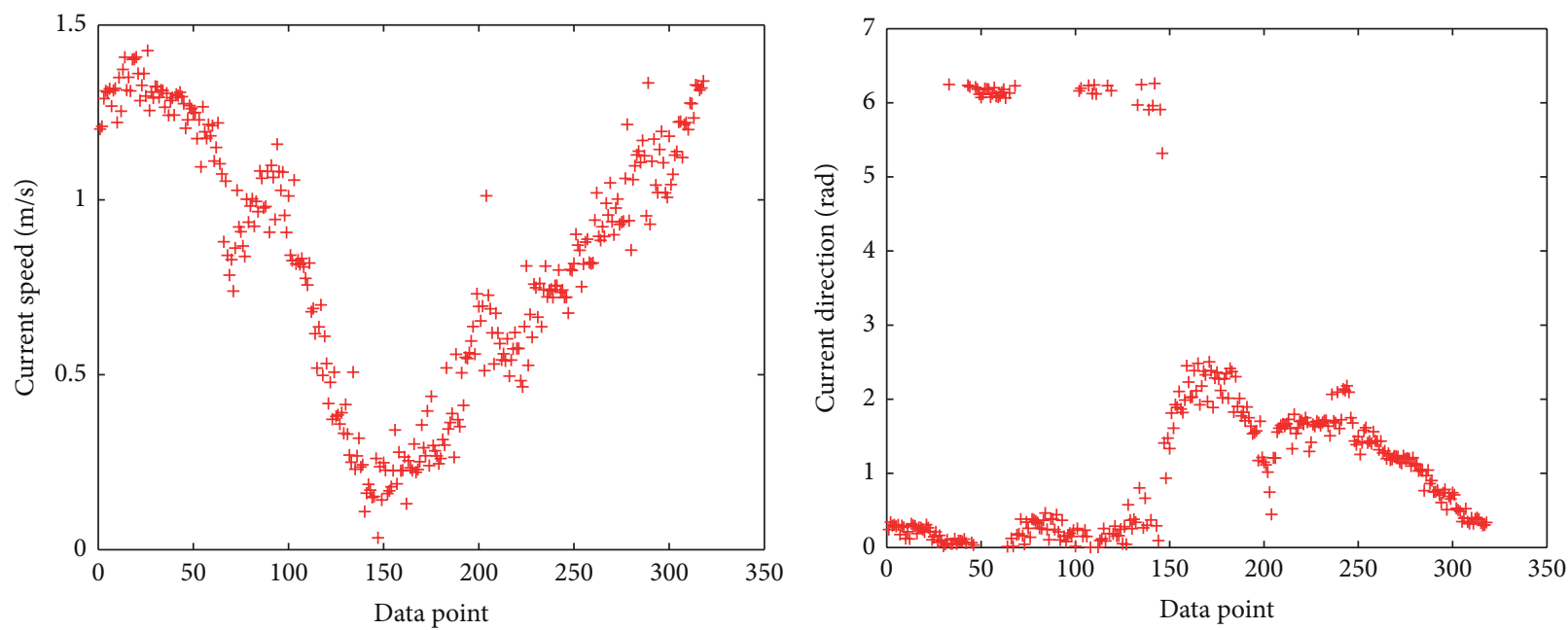

(c) $n=0.5$

FIGURE 9: Simulation results of different deviation order value. 
TABLE 6: Evaluation indicators of different observational data selection methods.

\begin{tabular}{lccc}
\hline & Method & Current speed indicators & Current director indicators \\
\hline LSM & \multirow{2}{*}{150 points of maximum energy are selected } & 0.0937 & 0.8708 \\
PSO & \multirow{2}{*}{1000 points of maximum energy are selected } & 0.2002 & 0.5269 \\
\hline LSM & & 0.0887 & 0.6025 \\
PSO & \multirow{2}{*}{ Points higher than 1\% of the maximum energy are selected } & 0.0875 & 0.3556 \\
PSO & All points & 0.0947 & 0.2957 \\
LSM & & 0.0840 & 0.1908 \\
PSO & & 0.0284 & 0.2289 \\
\hline
\end{tabular}

TABLE 7: Evaluation indicators of different initial data selection methods.

\begin{tabular}{|c|c|c|c|c|c|}
\hline Method & Current speed indicators & Current direction indicators & $T_{\min }$ & $T_{\max }$ & $T_{\text {avg }}$ \\
\hline PSO (initialization at random) & 0.0842 & 0.1564 & 11 & 100 & 33.4 \\
\hline PSO (initial results reference LSM) & 0.0840 & 0.1908 & 11 & 100 & 27.5 \\
\hline
\end{tabular}

TABLE 8: Evaluation indicators of different deviation order values.

\begin{tabular}{lcc}
\hline Method & Current speed indicators & Current director indicators \\
\hline PSO $(n=2)$ & 0.1864 & 0.2521 \\
PSO $(n=1)$ & 0.0840 & 0.1908 \\
PSO $(n=0.5)$ & 0.0750 & 0.1614 \\
\hline
\end{tabular}

51109045, and 51379049, Postdoctoral Foundation of Heilongjiang under Grant no. LBH-Z10217, and Foundation of Central University nos. HEUCF041216 and HEUCFX41302.

\section{References}

[1] D. H. Nomiyama and T. Hirayama, "Evaluation of marine radar as an ocean-wave-field detector through full numerical simulation," Journal of Marine Science and Technology, vol. 8, no. 2, pp. 88-98, 2003.

[2] I. K. Somawirata, K. Uchimura, and G. Koutaki, "Image enlargement using pyramid window kernel based on local image data," International Journal of Innovative Computing, Information and Control, vol. 9, no. 12, pp. 4863-4874, 2013.

[3] S. D. Lin, Y. Liu, and Y. Jhu, "A robust image descriptor for human detection based on HoG and Weber's law," International Journal of Innovative Computing, Information and Control, vol. 9, no. 10, pp. 3887-3901, 2013.

[4] I. R. Young, W. Rosenthal, and F. Ziemer, "A three-dimensional analysis of marine radar images for the determination of ocean wave directionality and surface currents," Journal of Geophysical Research: Oceans, vol. 90, no. C1, pp. 1049-1059, 1985.

[5] C. M. Senet, J. Seemann, and F. Ziemer, "The near-surface current velocity determined from image sequences of the sea surface," IEEE Transactions on Geoscience and Remote Sensing, vol. 39, no. 3, pp. 492-505, 2001.

[6] R. Gangeskar, "Ocean current estimated from X-band radar sea surface images," IEEE Transactions on Geoscience and Remote Sensing, vol. 40, no. 4, pp. 783-792, 2002.

[7] Y. Tang, Research on key techniques of wave remote sensing based on marine radar [Ph.D. thesis], Harbin Engineering University, 2010.

[8] G. Yuan, R. Jia, Y. Dai, and Y. Li, "Research on the extraction algorithm of ocean surface current based on sea clutter image,"
Chinese Journal of Scientific Instrument, vol. 32, no. 8, pp. 18451850, 2011.

[9] D. E. Barrick, "The role of the gravity-wave dispersion relation in HF radar measurements of the sea surface," IEEE Journal of Oceanic Engineering, vol. 11, no. 2, pp. 286-292, 1986.

[10] A. S. Li Chade, "Approximate dispersion relations of wave and current interaction," Science and Technology Information of Hehai University, no. 3, pp. 108-113, 1988.

[11] Z. Wang and M. Li, "Determine the wavelength with the wave dispersion relations," in Proceedings of the 16th National Seminar on Hydrodynamics, pp. 729-734, 2002.

[12] J. C. Nieto Borge and C. Guedes Soares, "Analysis of directional wave fields using X-band navigation radar," Coastal Engineering, vol. 40, no. 4, pp. 375-391, 2000.

[13] J. Wang, Improvement and application of particle swarm optimization algorithm [Ph.D. thesis], Northeastern University, 2006.

[14] L. Liu, Z. Fan, and C. Tao, "Research of X-band radar sea clutter image simulation method," in Proceedings of the 5th International Joint Conference on Computational Sciences and Optimization, pp. 793-796, 2012. 


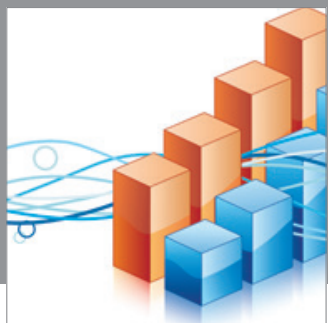

Advances in

Operations Research

mansans

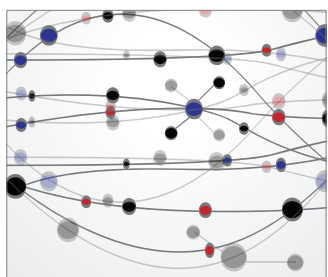

The Scientific World Journal
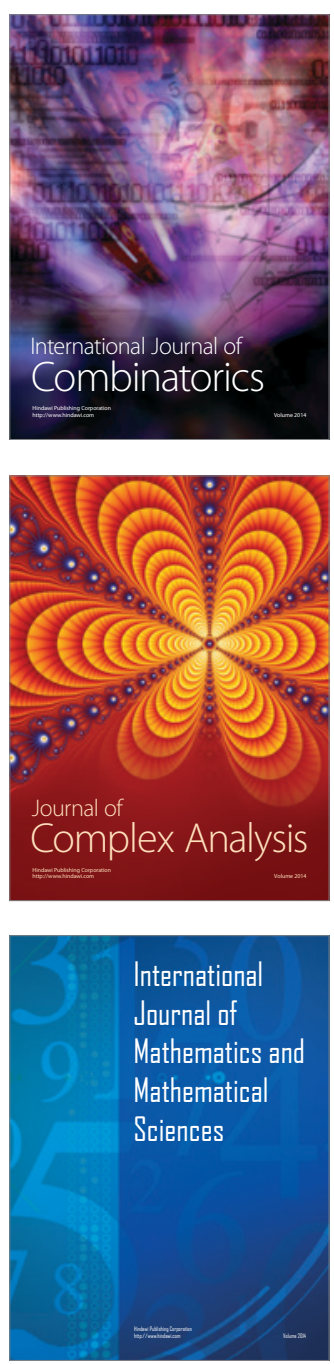
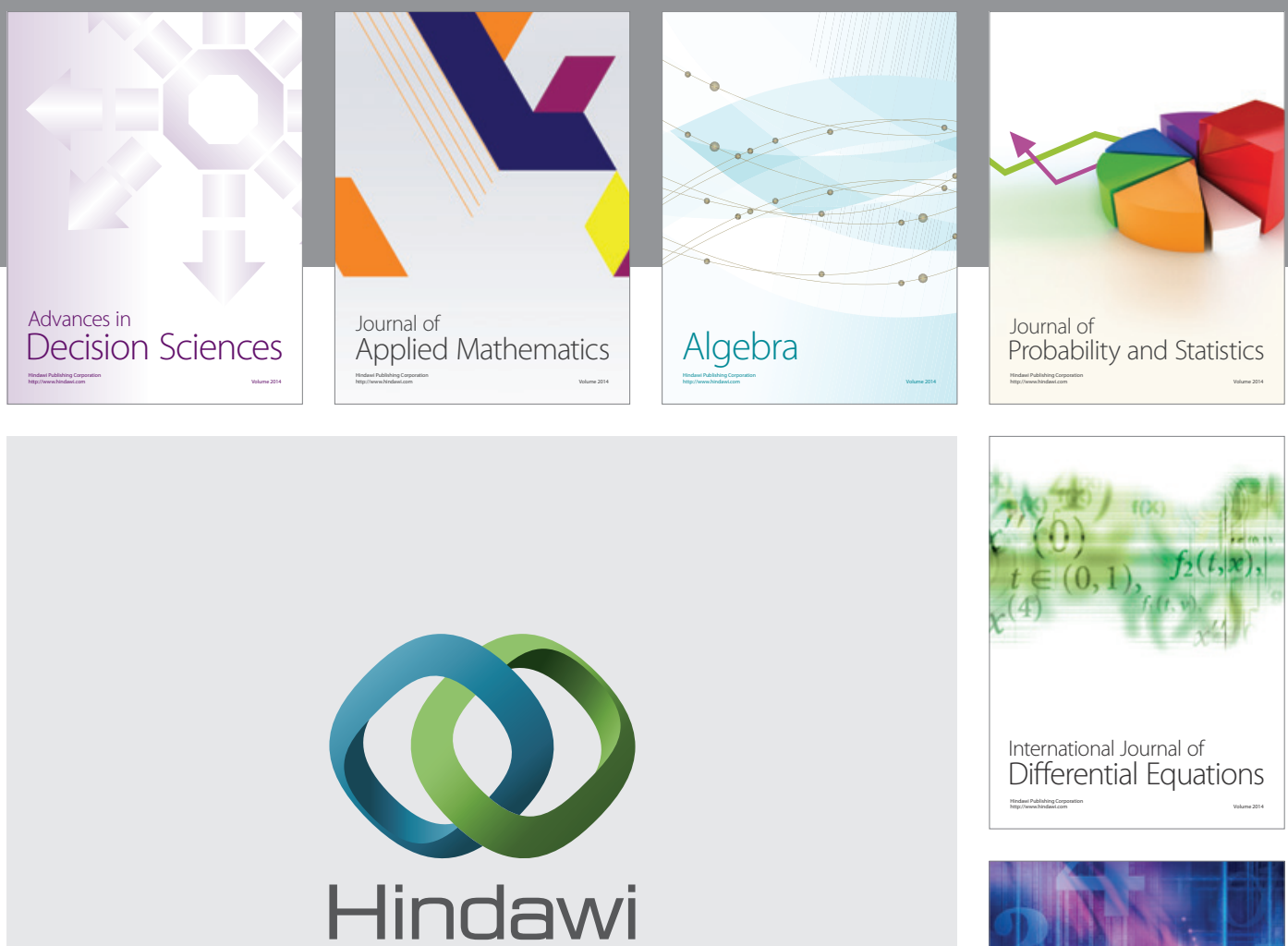

Submit your manuscripts at http://www.hindawi.com
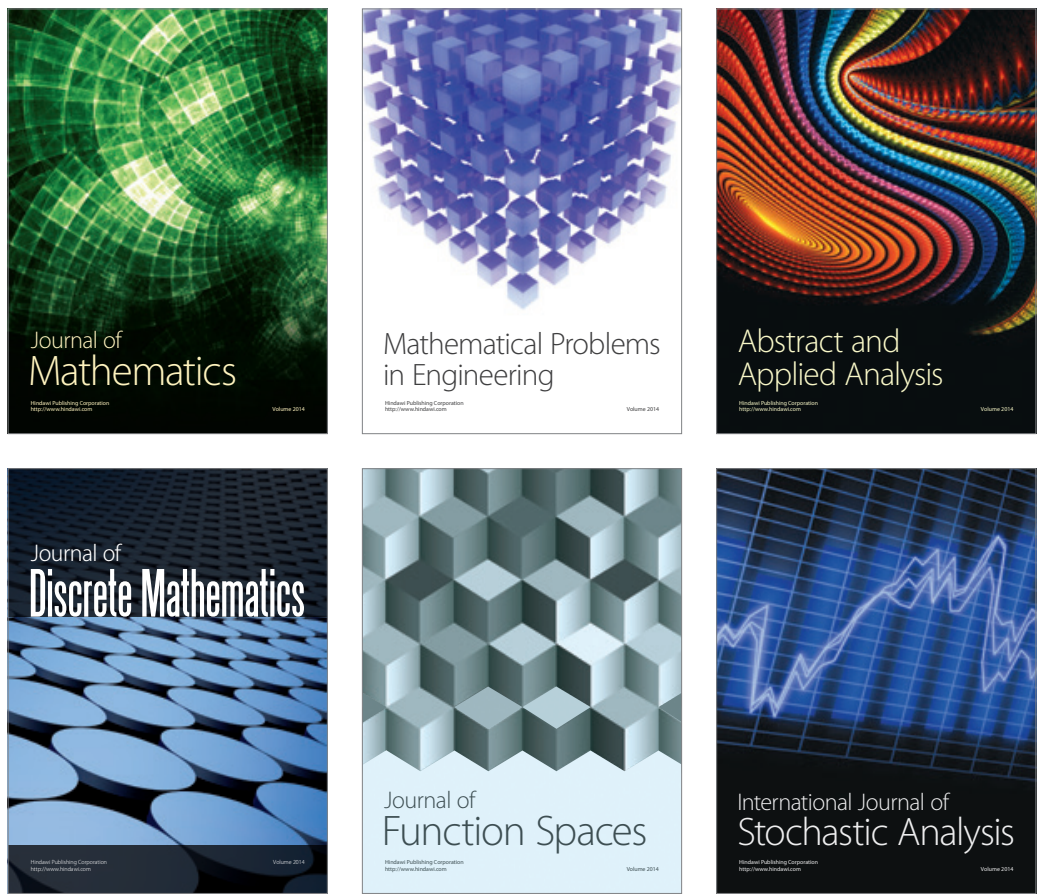

Journal of

Function Spaces

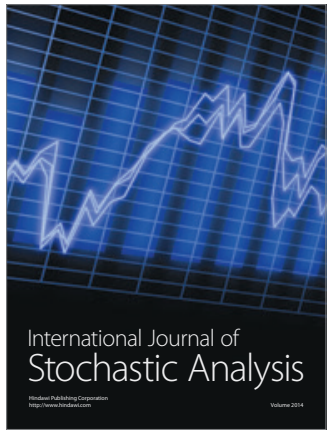

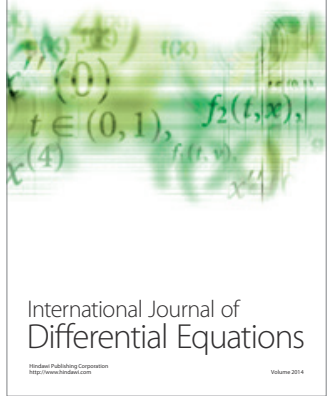
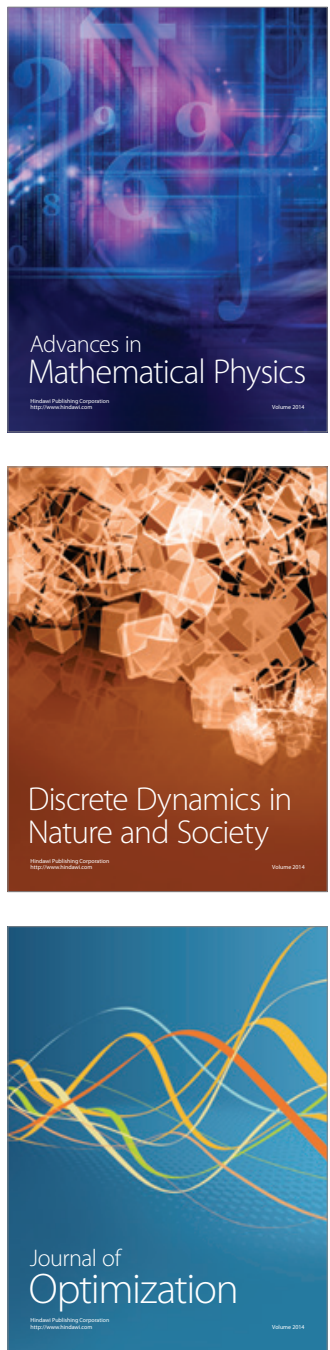